\title{
Effects of handedness on olfactory event-related potentials in a simple olfactory task*
}

Marie Gottschlich and Thomas Hummel

Smell \& Taste Clinic, Department of Otorhinolaryngology, TU Dresden, Dresden, Germany
Rhinology 53: 149-153, 2015

DOI:10.4193/Rhino14.204

*Received for publication:

August 25, 2014

Accepted: September 21, 2014

\begin{abstract}
The purpose of the present study was to re-investigate the influence of handedness on simple olfactory tasks to further clarify the role of handedness in chemical senses. Similar to language and other sensory systems, effects of handedness should be expected. Young, healthy subjects participated in this study, including 24 left-handers and 24 right-handers, with no indication of any major nasal or health problems. The two groups did not differ in terms of sex and age ( 14 women and 10 men in each group). They had a mean age of 24.0 years. Olfactory event-related potentials were recorded after left or right olfactory stimulation with the rose-like odor phenyl ethyl alcohol (PEA) or the smell of rotten eggs (hydrogen sulfide, $\mathrm{H}_{2} \mathrm{~S}$ ). Results suggested that handedness has no major influence on amplitude or latency of olfactory event-related potentials when it comes to simple olfactory tasks.
\end{abstract}

Key words: nose, smell, olfaction, olfactory event-related potentials, lateralization, handedness

\section{Introduction}

In contrast to other sensory systems such as vision or audition olfaction seems to be organized ipsilaterally ${ }^{(1-3)}$. However, preliminary works showed different results. Whereas the findings of Olofsson et al. ${ }^{(4)}$ suggest a general parietal, left-hemispheric predominance of response amplitudes to odorous stimulation, Brand and Jacquot ${ }^{(5)}$ observed a predominance of the right hemisphere to olfactory stimulation. According to the predominance of language, and in analogy to other sensory systems, effects of handedness should be expected (e.g., ${ }^{(6)}$ ). Although the left hemispheric dominance for language is well-known, the incidence of atypical language dominance increases linearly with the degree of left-handedness ${ }^{(7,8)}$. In contrast to that, no relationship between handedness and "eyedness" could be found (9). Concerning olfaction, data are inconsistent. For example, Hummel et al. ${ }^{(10)}$ showed no difference in odour thresholds in relation to handedness. Even so, in odour discrimination lefthanders performed significantly better at the left nostril compared with the right nostril, which was reversed in right-handers. These findings are contrary to what Zatorre et al. reported ${ }^{\text {(11). }}$ They found no influence of handedness or sex, but showed a general right-nostril advantage in odour discrimination. The objective of the present study was to re-investigate the influence of the subjects' handedness on simple olfactory tasks by recording olfactory event-related potentials obtained during unilateral olfactory stimulation.

\section{Material and Methods}

Subjects

Forty-eight healthy subjects participated in this study, including 24 (14 female) left-handers LH and 24 (14 female) right-handers RH. Handedness was assessed by the Edinburgh Handedness Inventory ${ }^{(12)}$. Participants had a mean age of 24.0 years (SD $=4.3$, range 18-45 years) and left-handed $(M=24.3, S D=5.0$ years) and right-handed ( $\mathrm{M}=23.8, \mathrm{SD}=3.5$ years) groups did not differ in age $(p=0.36)$. Exclusion criteria were neurological, psychiatric, endocrine or immunological diseases, diseases related to the upper respiratory tract, major septal deviations or a history of chronic medication. Using the "Sniffin' Sticks" odour identification test kit ${ }^{(13,14)}$ normal olfactory function was verified in all subjects, with no significant difference between $\mathrm{LH}$ and $\mathrm{RH}$. They were asked not to eat, drink or smoke for at least one hour prior to testing.

The study was performed according to the principles of the Helsinki declaration. The design was approved by the Ethics Committee of the Medical Faculty at the TU Dresden. 


\section{Stimulus presentation}

In two consecutive sessions of randomized order the two odorants phenyl ethyl alcohol (PEA: $40 \% \mathrm{v} / \mathrm{v}$, rose-like odour) and hydrogen sulfide $\left(\mathrm{H}_{2} \mathrm{~S}: 4 \mathrm{ppm}\right.$, smell of rotten eggs) were presented to the participants. Subjects participated in 3 sessions. During the first session participants were acquainted with the recording conditions. In the following two sessions either PEA or $\mathrm{H}_{2} \mathrm{~S}$ were used (sequence randomized across all subjects), each odorant was presented 32 times in randomized order to the left or right nostril.

For stimulation a computer-controlled air-dilution olfactometer (OM6b; Burghart, Wedel, Germany) was used. Odour pulses embedded in a constant flow of odorless air ( $6 \mathrm{~L}$ per min/nostril) were presented intranasally using TeflonTM tubing of $4 \mathrm{~mm}$ inner diameter. To avoid any additional stimulation, the air was humidified ( $80 \%$ relative humidity) and thermostabilized $\left(36^{\circ} \mathrm{C}\right)$. Stimulus duration was $200 \mathrm{~ms}$, and the interstimulus interval was 30s.

At the end of the sessions, subjects had to rate intensity and pleasantness of each stimulus on a visual analogue scale (VAS). Intensity was rated from 0 to 10 with higher ratings referring to greater perceived intensity. In hedonic ratings negative numbers $(\leq 0$ to -5$)$ indicated unpleasant sensations and positive numbers $(>0$ to +5$)$ indicated pleasant sensations. Participants were seated in a comfortable chair placed in an air-conditioned room. Before the testing session, they were trained in the breathing technique of velopharyngeal closure to maintain a constant airflow through the nasal cavity by lifting the soft palate ${ }^{(15,16)}$. In addition to that stable environmental conditions were created using white noise of 60-70 dB SPL applied over headphones and a tracking task on a video monitor, where a small square controlled by a joystick had to be kept inside a larger one that moved in an unpredictable pattern across the screen ${ }^{(17)}$. In a specific adaptation session, subjects were acquainted to these experimental conditions which optimized recording conditions. The experimenter (MG) was the same during the whole study.

\section{Electrophysiological recordings}

Olfactory event-related potentials (OERP) were recorded at 5 positions of the scalp according to the 10/20 international system of electrode placement $(\mathrm{Cz}, \mathrm{Fz}, \mathrm{Pz}, \mathrm{C} 3, \mathrm{C} 4)$, referenced to linked earlobes $(A 1+A 2)$. Eye blinks were monitored at position Fp2; if necessary artifact-contaminated records were rejected. Neuro-electrical activity was recorded for 2048 ms (including 500 ms pre-stimulus period) using an 8-channel amplifier (Schubert, Röttenbach, Germany). The sampling frequency was 250 $\mathrm{Hz}$ (band pass $0.2-30 \mathrm{~Hz}$ ) Using the program EPEvaluate (Kobal, Erlangen, Germany) a minimum of $6(7 \pm 1)$ records without artifacts for each odorant and stimulation side was averaged offline ${ }^{(16)}$ with a low-pass of $15 \mathrm{~Hz}$. Then amplitudes and latencies were measured by a trained observer (MG): amplitudes - ampN1, ampP2; peak-to-peak amplitudes - ptpN1P2; latencies - latN1, latP2.

\section{Statistical analyses}

Statistical analyses were performed using SPSS 21.0 for Windows (SPSS Inc., Chicago, IL, USA) and submitted to an analysis of variance for repeated measures (rm-ANOVA) with the factors "odorant" (PEA, H2S), "presentation side" (left nostril, right nostril) and "recording position" ( $\mathrm{Cz}, \mathrm{Fz}, \mathrm{Pz}, \mathrm{C} 3, \mathrm{C} 4)$ as within-subject factors and "handedness" (LH, RH) as a between-subject factor. Degrees of freedom were corrected by the Greenhouse-Geisser procedure. The significance level was set at $p<0.05$. Due to the relatively small sample size only main effects or two-way interactions were interpreted. Some subjects had to be excluded (e.g., because there were no detectable responses at some of the recording sites $\left.{ }^{(18)}\right)$, so that complete datasets of 18 left-handers and 14 right-handers could be used for statistical analyses.

\section{Results}

\section{Psychophysical data}

Psychophysical data (Table 1) revealed no difference between the intensity of the two odours $(t=1.42, p=0.16)$. The data clearly show that $\mathrm{H}_{2} \mathrm{~S}$ was rated more unpleasant than PEA ( $\mathrm{t}=$ $12.6, p<0.001)$. However, there were no significant differences depending on handedness $(t<0.93, p>0.36)$.

\section{Olfactory event-related potentials}

Statistical analyses did not reveal a main effect of the factor "handedness". Only for latN1 an interaction between factors "handedness" and "odorant" was present $(F[1,30]=5.21, \mathrm{p}=$ $0.03)$, indicating that in general, $\mathrm{LH}$ compared to $\mathrm{RH}$, had shorter peak latencies for PEA, whereas this was the other way around

Table 1. Descriptive statistics of ratings (means, standard deviations), separately for the subjects' handedness ( $L H-n=24, R H-n=24$ ).

\begin{tabular}{|llccc|}
\hline PEA $(\mathrm{n}=24)$ & Pleasantness & LH & 1.71 & 2.58 \\
& & $\mathrm{RH}$ & 1.38 & 1.58 \\
\hline & Intensity & LH & 3.04 & 1.90 \\
\hline $\mathrm{H}_{2} \mathrm{~S}(\mathrm{n}=24)$ & Pleasantness & $\mathrm{LH}$ & 3.49 & 1.46 \\
& & $\mathrm{RH}$ & -2.83 & 1.76 \\
& & $\mathrm{RH}$ & 1.61 \\
& Intensity & $\mathrm{LH}$ & 2.89 & 1.40 \\
& & $\mathrm{RH}$ & 3.17 & 1.11 \\
\hline
\end{tabular}


Table 2. Descriptive statistics of olfactory ERP, separately for side of stimulation and the subjects' handedness $(\mathrm{LH}-\mathrm{n}=18, \mathrm{RH}-\mathrm{n}=14)$.

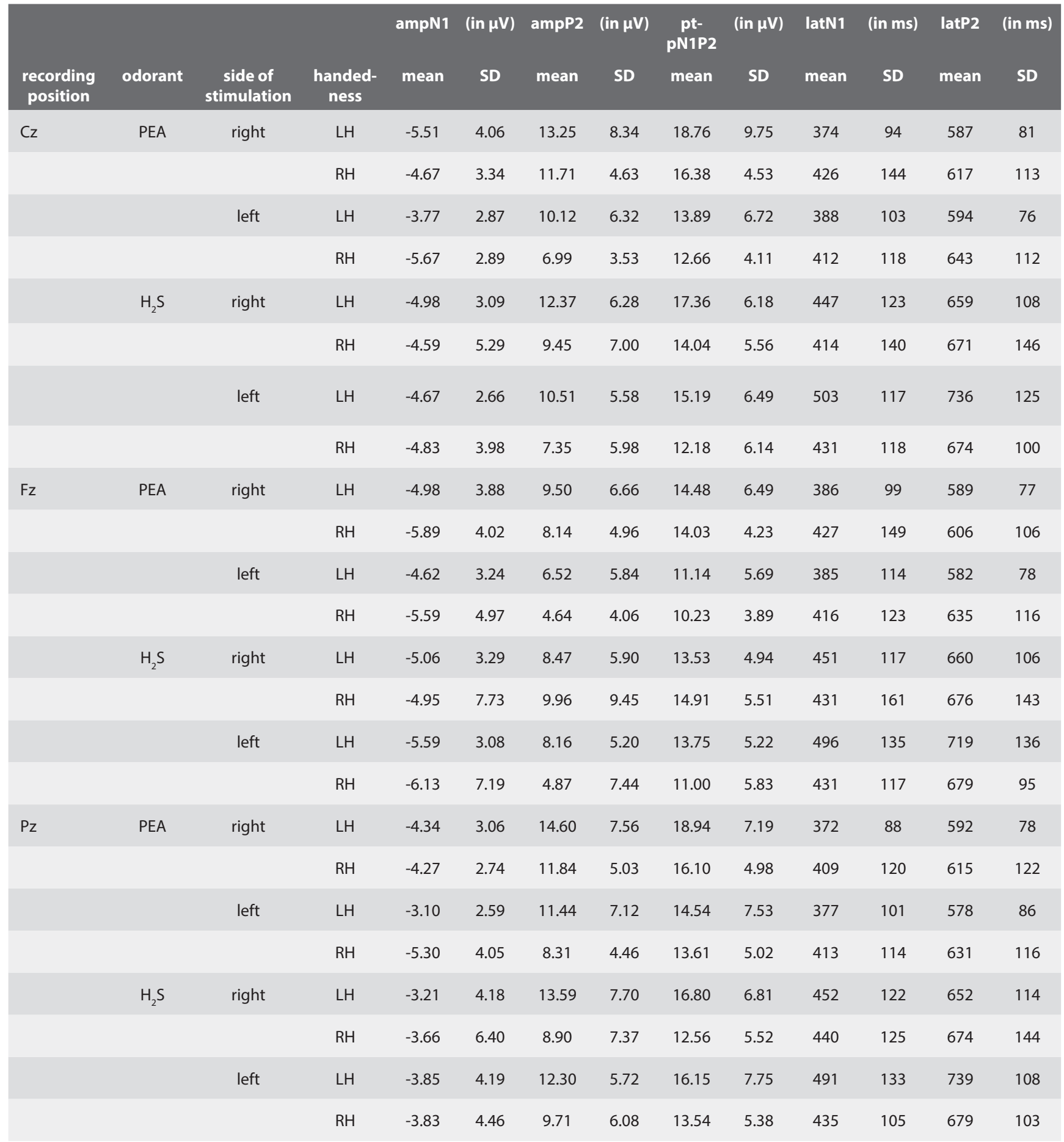

for $\mathrm{H}_{2} \mathrm{~S}$ (Table 2).

Regarding the factor "presentation side" a significant main effect emerged for ptpN1P2 $(F[1,30]=5.39, p=0.027)$ with larger response amplitudes for stimulation of the right nostril.

The factor "odorant" was significant for latN1 $(F[1,30]=9.22, p=$ 0.005 ) with shorter latencies for PEA compared to $\mathrm{H}_{2} \mathrm{~S}$. Similar, but yet even more pronounced findings were made for latP2 (factor "odorant": $F[1,30]=23.3, p<0.001$ ). A significant interaction "odorant" * "position" was observed for ptpN1P2 (F[4,120] = $3.07, p=0.019)$; this finding was based on a more even distribution of response amplitudes to $\mathrm{H}_{2} \mathrm{~S}$ across the skull, whereas PEA produced more pronounced differences with largest midline response at $\mathrm{Pz}$ and higher amplitudes at $\mathrm{C} 4$.

The factor "recording position" was not significant for latencies, 
but for ampN1 $(F[4,120]=6.01, p<0.001)$, ampP2 $(F[4,120]=$ $35.8, p<0.001)$, and for $\operatorname{ptpP} 2(F[4,120]=23.5, p<0.001)$. In all of these cases response amplitudes were largest at centro-parietal sites and larger at the right than at the left side ${ }^{(19-21)}$.

\section{Discussion}

With regard to possible effects of handedness on the early processing of olfactory information, no main effect of the factor "handedness" became significant indicating that handedness has no major effect on the processing of olfactory information. However, results indicated that ERP peak latencies N1 were shorter in $\mathrm{LH}$ compared to $\mathrm{RH}$ for the pleasant rose-like odour PEA, whereas this was the other way around for the unpleasant rotten egg-like smell of $\mathrm{H}_{2} \mathrm{~S}$. It may be speculated that this result might reflect hemispheric specialization in terms of the processing of valence ${ }^{(19)}$. However, this hypothesis would need to be corroborated in a different study using a larger number of odours.

As already indicated in the Introduction, previously published results are not very homogeneous. Some work suggests that odour discrimination is best at the left side in lefthanders and vice versa ${ }^{(10)}$, while other studies suggest that handedness does not affect this task ${ }^{(22)}$. For detection thresholds conflicting results have been shown with right-handers showing higher sensitivity on the left side of the nose whereas this was the other way around in left-handers ${ }^{(23)}$; the contrary has been reported by others, although in smaller sample sizes ${ }^{(24)}$. Yet other studies did not report significant effects of handedness on odour thresholds ${ }^{(22,25)}$. In addition, other tasks like odour memory ${ }^{(26)}$ had no simple effect on odour processing (see also $\left.{ }^{(27)}\right)$. Similar findings have been reported previously for passive olfactory tasks ${ }^{(28,29)}$. Some authors showed significant effects of handedness in FMRI activations, which was not found by others ${ }^{(28)}$. Thus, the current results contribute to the idea that handedness does not play a major role in the perception of odours, at least not with regard to relatively simple testing environments.

Interestingly, the side of stimulation had a significant effect on ERP amplitudes with larger responses when the right nostril was stimulated. Considering ipsilateral processing of olfactory information ${ }^{(2)}$, this may be interpreted such that the right side is more significant for olfaction than the left side. This idea is supported by other studies using olfactory ERP (1), magnetoencephalography ${ }^{(30)}$ and other studies ${ }^{(31)}$. However, lateralized processing appears to be largely task-dependent ${ }^{(32,33)}$.

In conclusion, the prominent finding of the present investigation was that handedness had no major effect on ratings of odours or on amplitudes or latencies of olfactory ERP obtained in young healthy people, using passive odour presentation.

\section{Acknowledgement}

This research did not receive external funding.

\section{Authorship contribution}

MB: designed study, conducted experiments, analysed data, wrote manuscript; $\mathrm{TH}$ : designed study, conducted experiments, analysed data, wrote manuscript.

\section{Conflicts of interest}

None of the authors declares a conflict of interest.

\section{References}

1. Hummel T, Pauli E, Schuler P, Kettenmann B, Stefan H, Kobal G. Chemosensory eventrelated potentials in patients with tempora lobe epilepsy. Epilepsia. 1995; 36: 79-85.

2. Lascano AM, Hummel T, Lacroix JS, Landis BN, Michel CM. Spatio-temporal dynamics of olfactory processing in the human brain: an event-related source imaging study. Neuroscience. 2010; 167: 700-708.

3. Fontaine CJ, Harley CW, Yuan Q. Lateralized Odor Preference Training in Rat Pups Reveals an Enhanced Network Response in Anterior Piriform Cortex to Olfactory Input That Parallels Extended Memory. J Neurosci .2013; 33: 5126-15131.

4. Olofsson JK, Broman DA, Gilbert PE, Dean $P$, Nordin S, Murphy C. Laterality of the olfactory event-related potential response. Chem Senses. 2006, 31: 699-704.

5. Su N, Ching V, Grushka M. Taste Disorders: A Review. J Can Dent Assoc. 2013; 79:d86.

6. Frasnelli J, Hummel T. Olfactory dysfunction and daily life. Eur Arch Otorhinolaryngol. 2005, 262: 231-235.

7. Schriever VA, Lehmann S, Prange J, Hummel T. Preventing olfactory deterioration: olfactory training may be of help in older people. J Am Geriatr Soc. 2014, 62: 384-386.

8. Chen AW, Resurreccion AV. Age appropriate hedonic scales to measure food preferences of young children. Journal of Sensory Studies 1996, 11:141-163

9. Seyal M, Sato S, White BG, Porter RJ. Visual evoked potentials and eye dominance. Electroencephalogr Clin Neurophysiol 1981, 52:424-428.

10. Hummel T, Mohammadian P, Kobal G Handedness is a determining factor in lateralized olfactory discrimination. Chemical Senses. 1998, 23: 541-544.

11. Zatorre RJ, Jones-Gotman M. Human olfactory discrimination after unilateral frontal or temporal lobectomy. Brain. 1991, 114 71-84.

12. Oldfield RC. The assessment and analysis of handedness: the Edinburgh inventory. Neuropsychologia. 1971, 9: 97-113.

13. Kobal G, Hummel T, Sekinger B, Barz S, Roscher S, Wolf SR. "Sniffin'Sticks": Screening of olfactory performance. Rhinology. 1996, 34: 222-226

14. Hummel T, Kobal G, Gudziol H, Mackay-Sim A. Normative data for the "Sniffin' Sticks" including tests of odor identification, odor discrimination, and olfactory thresholds: an upgrade based on a group of more than 3,000 subjects. Eur Arch Otorhinolaryngol. 2007, 264: 237-243.

15. Nagel WA. Einige Bemerkungen über nasales Schmecken. Ztschr f Psychol. 1904, 25: 268.

16. Kobal G. Elektrophysiologische Untersuchungen des menschlichen Geruchssinns. Stuttgart, Thieme Verlag, 1981.

17. Hummel T, Kobal G. Olfactory event-related potentials. In: Simon SA, Nicolelis MAL, es. Boca Raton, Florida, USA, CRC press, 2001, 
p. pp. 429-464.

18. Lotsch J, Hummel T. The clinical significance of electrophysiological measures of olfactory function. Behav Brain Res. 2006, 170: 78-83.

19. Kobal G, Hummel T, Van Toller S. Differences in chemosensory evoked potentials to olfactory and somatosensory chemical stimuli presented to left and right nostrils. Chem Senses. 1992, 17: 233-244.

20. Hummel T, Livermore A, Hummel C, Koba G. Chemosensory event-related potentials in man: relation to olfactory and painful sensations elicited by nicotine. Electroencephalogr Clin Neurophysiol. 1992, 84: 192-195.

21. Hummel T, Kobal G. Differences in human evoked potentials related to olfactory or trigeminal chemosensory activation. Electroenceph Clin Neurophysiol. 1992 84:.84-89

22. Zatorre RJ, Jones-Gotman M. Right-nostril advantage for discrimination of odors Percept Psychophys. 1990, 47: 526-531.

23. Frye RE, Doty RL, Shaman P. Bilateral and unilateral olfactory sensitivity: relationship to handedness and gender. In: Doty RL, Müller-Schwarze D, ed. New York, Plenum Press, 1992, p. pp. 559-564.

24. Youngentob SL, Kurtz DB, Leopold DA,
Mozell MM, Hornung DE. Olfactory sensitivity: is there laterality? Chem Senses. 1982, 7: 1-9.

25. Betchen SA, Doty RL. Bilateral detection thresholds in dextrals and sinistrals reflect the more sensitive side of the nose, which is not lateralized. Chem Senses. 1998, 23 453-457.

26. Doty RL, Kerr KL. Episodic odor memory: influences of handedness, sex, and side of nose. Neuropsychologia. 2005, 43:17491753.

27. Gilbert AN, Crouch M, Kemp SE. Olfactory and visual mental imagery. J Mental Imagery. 1998, 22: 137-146.

28. Lübke K, Gottschlich M, Gerber JC, Pause BM, Hummel T. No effects of handedness on passive processing of olfactory stimuli: a FMRI study. Chemosens Percept. 2012, 5: 22-26.

29. Hebbal GV, Mysorekar VR. Anatomical and behavioural asymmetries in right and left handers from India. Ann Anat. 2003, 185: 267-275.

30. Kettenmann B, Hummel C, Stefan H, Kobal G. Multiple olfactory activity in the human neocortex identified by magnetic source imaging. Chem Senses. 1997, 22: 493-502.

31. Hudry J, Ryvlin P, Saive AL, Ravel N, Plailly J, Royet JP. Lateralization of olfactory pro- cessing: Differential impact of right and left temporal lobe epilepsies. Epilepsy Behav. 2014, 37C: 184-190.

32. Royet JP, Plailly J. Lateralization of olfactory processes, Chem Senses. 2004, 29: 731-745.

33. Doty RL, Bromley SM, Moberg PJ, Hummel T. Laterality in human nasal chemoreception. In: Christman S, ed. Amsterdam, North Holland Publishing, 1997, p. pp. 497-542.

\section{Thomas Hummel}

Smell \& Taste Clinic

Department of Otorhinolaryngology

TU Dresden

Fetscherstrasse 74

01307 Dresden

Germany

Tel: +49-351-458-4189

E-mail: thummel@mail.zih.tu-dres-

den.de 